\title{
0X40L/0X40 axis impairs follicular and natural Treg function in human SLE
}

\author{
Clément Jacquemin, ${ }^{1}$ Jean-François Augusto,, ${ }^{2}$ Marc Scherlinger, ${ }^{2,3}$ Noémie Gensous, ${ }^{2}$ \\ Edouard Forcade, ${ }^{2}$ Isabelle Douchet, ${ }^{2}$ Emeline Levionnois, ${ }^{2}$ Christophe Richez, ${ }^{2,3}$ Estibaliz Lazaro, ${ }^{2,3}$ \\ Pierre Duffau, ${ }^{2,3}$ Marie-Elise Truchetet, ${ }^{2,3}$ Julien Seneschal,, ${ }^{1,3}$ Lionel Couzi, ${ }^{3}$ Jean-Luc Pellegrin, ${ }^{3}$ \\ Jean-François Viallard, ${ }^{3}$ Thierry Schaeverbeke, ${ }^{3}$ Virginia Pascual, ${ }^{4}$ Cécile Contin-Bordes,, 3 \\ and Patrick Blanco ${ }^{2,3}$
}

IINSERM U1035, Immuno-Dermatology, Bordeaux University, Bordeaux, France. ²CNRS-UMR 5164 Immuno ConcEpT, Bordeaux University, Bordeaux, France. ${ }^{3}$ Centre hospitalier universitaire de Bordeaux, Bordeaux, France. ${ }^{4}$ Baylor Institute for Immunology Research, Dallas, Texas, USA.

Tregs are impaired in human systemic lupus erythematosus (SLE) and contribute to effector T cell activation. However, the mechanisms responsible for the Treg deficiency in SLE remain unclear. We hypothesized that the OX40L/OX40 axis is implicated in Treg and regulatory follicular helper T (Tfr) cell dysfunction in human SLE. OX40L/OX40 axis engagement on Tregs and Tfr cells not only specifically impaired their ability to regulate effector $\mathrm{T}$ cell proliferation, but also their ability to suppress T follicular helper (Tfh) cell-dependent B cell activation and immunoglobulin secretion. Antigen-presenting cells from patients with active SLE mediated Treg dysfunction in an OX40Ldependent manner, and OX40L-expressing cells colocalized with Foxp $3^{+}$cells in active SLE skin lesions. Engagement of the $0 \times 40 \mathrm{~L} / 0 \times 40$ axis resulted in Foxp3 downregulation in Tregs, and expression in SLE Tregs correlated with the proportion of circulating OX40L-expressing myeloid DCs. These data support that OX40L/OX40 signals are implicated in Treg dysfunction in human SLE. Thus, blocking the $0 \times 40 \mathrm{~L} / 0 \times 40$ axis appears to be a promising therapeutic strategy.

Authorship note: C] and JFA are co-first authors

Conflict of interest: The authors have declared that no conflict of interest exists.

License: Copyright 2018, American Society for Clinical Investigation.

Submitted: May 11, 2018 Accepted: November 14, 2018 Published: December 20, 2018

\section{Reference information:} JCI Insight. 2018;3(24):e122167. https://doi.org/10.1172/jci. insight.122167.

\section{Introduction}

Systemic lupus erythematosus (SLE) is an autoimmune disease characterized by a loss of tolerance toward nuclear components, leading to autoantibody production, immune complex formation, and organ/tissue damages (1). There is a strong need for a better understanding of human SLE pathogenesis, as only a few drugs are effective, and only one has been approved for lupus treatment over the past 50 years (2). The precise immunological events that trigger the onset of SLE remain unknown, but the established chronic innate activation of the DC system plays a critical role in the activation of autoreactive $\mathrm{T}$ and $\mathrm{B}$ lymphocytes while overwhelming natural regulatory mechanisms $(3,4)$.

Foxp ${ }^{+}$Tregs are important for the maintenance of immunological tolerance, and their absence results in fatal autoimmune diseases in humans and mice (5). In SLE, defects in the Treg compartment have been recorded in both mouse models and in patients. Treg depletion 3 days after birth in the NZB $\times$ NZW F1 SLE mouse model is associated with the development of nephritis and an increased production of antiDNA antibodies, whereas adoptive transfer of purified and ex vivo-expanded Tregs slows the progression of renal disease when administered after the onset of proteinuria (6). In SLE patients, studies assessing Tregs have generated contradictory results regarding quantitative and qualitative aspects of these cells in this disease (7). Certain studies have reported reduced numbers or impaired function of circulating Tregs $(8,9)$, while other groups have not observed any impairment $(10)$. Moreover, some groups have even found increased levels of Tregs in comparison to levels found in healthy subjects $(11,12)$. Finally, the failure of Tregs to control exaggerated $\mathrm{T}$ cell and B cell activation in SLE may depend on cell-extrinsic factors, including the interplay of Tregs with other immune cells in an inflammatory milieu. Moreover, two groups described follicular regulatory $\mathrm{T}$ (Tfr) cells that express (Foxp3) and B cell lymphoma 6 (Bcl-6) and that have high expression of CXCR5, ICOS, and programmed cell death 1 (PD1). This population migrates to the B cell follicles and inhibits antibody production $(13,14)$. As SLE patients with active disease exhibit abnormalities in the peripheral B cell compartment, including intensive germinal center (GC) activity (15), 
studying the consequences of this population of cells and its mixed regulatory and follicular features is of particular interest in SLE.

Our group recently reported that OX40L was expressed by myeloid antigen-presenting cells (APCs), both in blood and in inflamed tissues of adult and pediatric SLE patients (16). The frequency of circulating OX40L-expressing myeloid APCs correlated with disease activity as well as with the frequency of activated blood follicular helper $\mathrm{T}$ (Tfh) cells. OX40L stimulation induced human $\mathrm{CD}^{+}{ }^{+} \mathrm{T}$ cells to express Tfh cellassociated molecules and was sufficient to induce memory and naive $\mathrm{CD} 4^{+} \mathrm{T}$ cells to become functional $\mathrm{B}$ cell helpers. Interestingly, besides this newly described role in promoting $\mathrm{Tfh}$ cell differentiation (17), OX40L has been shown to block Treg functions both in mice and in humans (18). Its role in the recently described Tfr cell function remains unknown (13). Thus, in the present study, we investigated the role of the OX40L/OX40 axis on the functions of both Tregs and Tfr cells in human SLE.

\section{Results}

Soluble and membrane-bound OX40L impair the suppressive functions of Tregs. We recently observed that soluble OX40L (sOX40L) strongly downregulated Treg-related genes, including Foxp3 and the IKZF2-encoding Helios gene, in naive and memory T cells stimulated with anti-CD3 and anti-CD28 after 48 hours of culture (16). This observation suggests that the OX40L/OX40 axis not only promotes Tfh cell differentiation of Th cells, but can also affect the generation and/or function of the Treg compartment. In order to analyze the effect of sOX40L on the Treg function, purified Tregs $\left(\mathrm{CD} 4^{+} \mathrm{CD} 25^{\mathrm{hi}} \mathrm{CD} 127^{-}\right)$were stimulated with recombinant sOX40L and cultured with CFSE-labeled healthy donor (HD) effector T4 (Eff.T4) cells $\left(\mathrm{CD} 4^{+} \mathrm{CD} 25^{-} \mathrm{CD} 127^{+}\right)$in the presence of anti-CD3 and anti-CD28. We observed that sOX40L decreased Treg-mediated suppression of Eff.T4 cell proliferation (Figure 1, A and B, $P=0.028$ ), without inducing Treg death. To evaluate whether membrane-bound OX40L was also capable of altering Treg function, we took advantage of the ability of anti-RNP ${ }^{+}$SLE sera to upregulate OX40L expression on HD monocytes (SLE DCs) (Supplemental Figure 1, C and D) $(3,16)$. Indeed, within circulating APCs, SLE CD11c DR ${ }^{+}$ DCs and monocytes (but not B cells) showed increased OX40L expression compared with that in HD DCs and monocytes (Supplemental Figure 1, E and F). Eff.T4 cells and Tregs were purified from blood of HDs and cultured along with DCs differentiated with GM-CSF and IL-4 (GM-CSF ${ }^{+}$IL-4 DCs) or SLE DCs. As compared with GM-CSF ${ }^{+}$IL-4 DCs, coculture with SLE DCs was associated with a substantial decrease of the ability Tregs to suppress Eff.T4 cell proliferation in a dose-dependent manner (Figure 1C). As a control, the SLE DC-dependent decrease of Treg function was maintained independently of the Eff. T4/Treg ratio (Figure 1D). This process was OX40L dependent, as Treg-suppressive function was restored when SLE DCs were preincubated with a blocking anti-OX40L mAb (Figure 1, E and F). Furthermore, OX40 costimulation did not alter the proliferation capacities of Eff.T4 (Supplemental Figure 2), supporting the hypothesis that OX40L acts on Treg functions. Altogether, these results demonstrate that both sOX40L and membrane-bound OX40L block the suppressive function of purified allogeneic FoxP $3^{+}$Tregs in vitro.

OX40L-expresssing APCS from patients with active SLE mediate Treg dysfunction. In order to confirm that an OX40L-dependent Treg dysfunction could operate in SLE patients, we monitored OX40L and OX40 expression in SLE patients. We observed that circulating monocytes from patients with active SLE expressed OX40L (Supplemental Figure 1, E and F) (16) and that SLE patients $(n=25)$ had a higher serum concentration of sOX40L than that in HDs $(n=15)$ (Supplemental Figure 3A). A positive correlation between sOX40L blood concentration and SLE Disease Activity Index (SLEDAI) was observed in SLE patients (Supplemental Figure 3B). Circulating Tregs from SLE patients had a higher expression of OX40 than those from HDs (Supplemental Figure 3, C and D, $P=0.0055$ ). To analyze the functional consequences of upregulated OX40L expression by monocytes on Tregs, we purified CD $14^{+} \mathrm{CD} 11 \mathrm{c}^{+} \mathrm{HLA}-\mathrm{DR}^{+} \mathrm{APC}$ from the blood of HDs and SLE patients and cultured them with purified allogeneic HD Eff.T4 cells in the presence or absence of Tregs (Supplemental Table 3). Figure 2A depicts a representative experiment showing the impairment of the Treg-suppressive function in the presence of APCs purified from SLE patient with an active disease. Blocking OX40L signaling with a blocking anti-OX40L mAb restored the Treg-suppressive function (Figure 2A). This observation was further confirmed with APCs purified from 5 patients with active SLE, as compared with APCs purified from $7 \mathrm{HDs}$ (mean percentage of inhibition, $25.5 \% \pm 22 \%$ vs. $72.9 \% \pm 9.6 \%$, respectively, $P=0.01$ ) (Figure $2 \mathrm{~B}$ ). APCs from 5 SLE patients with inactive disease only had a minor effect on Treg-suppressive function, and anti-OX40L mAb had no significant effect (Figure 2B). Conversely, we observed an inverse correlation between OX40L expression on SLE APCs and their ability 
to hamper Treg-suppressive function (Figure $2 \mathrm{C}, \mathrm{r}=-0.85, P=0.0001$ ). In order to see whether the interaction between OX40L ${ }^{+}$cells and Tregs was plausible in vivo, we stained skin biopsies from SLE patients with OX40L and Foxp3. We observed a close proximity between infiltrating Foxp3 ${ }^{+}$cells and $\mathrm{OX}_{40 \mathrm{~L}^{+}}$cells in affected skin biopsies from SLE patients, suggesting that $\mathrm{OX} 40 \mathrm{~L}^{+}$cell-Treg contact actually operates in vivo within inflammatory tissues (Figure 2D). Altogether, these data suggest that APCs from patients with active SLE can suppress the function of Foxp3 ${ }^{+}$Tregs in an OX40L-dependent manner.

OX40L-expressing APCs do not confer Treg resistance to effector $T$ cells. The OX40L-dependent decrease of Eff.T4 cell proliferation in the coculture experiments may be the consequence of either an impaired suppressive function of Tregs or an Eff.T4 cell resistance to Treg-mediated suppression (19). In order to evaluate whether SLE DCs could promote Eff.T4 cells to become resistant to Treg suppression, we set up a 3-day coculture of GM-CSF ${ }^{+}$IL-4 DCs or SLE DCs with purified allogeneic Eff.T4 cells. Eff.T4 cells were then purified and further activated and cultured with HD-purified Tregs and GM-CSF ${ }^{+}$IL-4 DCs (schematic of the experiment shown in Supplemental Figure 4). As shown in Figure 3A, suppression of Eff.T4 cell proliferation was equivalent, regardless of the origin of Eff.T4 cells (GM-CSF IL-4- or SLE DC culture), demonstrating that OX40L signals provided by SLE DCs to Eff.T4 cells did not render these cells (the Eff.T4 cell) resistant to Tree-mediated suppression. Moreover, CD4 ${ }^{+} \mathrm{T}$ cell expression of TNF receptor II (TNFR2), a marker that has been previously shown to be associated with Eff.T4 cell resistance to Treg suppression (20), as well as the concentration of TNF- $\alpha$ in coculture supernatant (data not shown) were equivalent in both GM-CSF ${ }^{+}$IL-4 DC and SLE DC culture conditions (Figure 3, B and C). Therefore, these data support that impaired Treg suppression is the consequence of direct Tregs OX40L-mediated signaling.

OX40L-expressing SLE DCs modulate Treg function through downregulation of Foxp3 expression. In order to assess the underlying mechanisms involved in OX40L-mediated Treg dysfunction, we next analyzed the expression of Treg-associated surface markers and intranuclear transcription factors, including CD25, cytotoxic T lymphocyte antigen-4 (CTLA-4), glucocorticoid-induced tumor necrosis factor receptor (GITR), and Foxp3, in Tregs cocultured with GM-CSF ${ }^{+}$IL-4 DCs or SLE DCs and Eff.T4 cells. As shown in Figure 4A, the expression of CD25, CTLA-4, and GITR was similar in Tregs cultured under different conditions, whereas intranuclear Foxp3 expression was significantly lower in SLE DC compared with GM-CSF ${ }^{+}$IL-4 DC conditions $(P=0.02)$ (Figure 4B). Moreover, Foxp3 downregulation was inhibited by the addition of a blocking anti-OX40L mAb $(P=0.03)$ (Figure 4B). OX40L-mediated Foxp3 downregulation was further confirmed in Tregs cultured in presence of sOX40L, murine fibroblasts transfected with human OX40L (Figure 4, C and D, respectively), and in the different subsets of Tregs (resting and active Tregs) (Supplemental Figure 3E). Foxp3 downregulation was associated in vitro with an OX40L-dependent Helios downregulation as well (Supplemental Figure 5). We measured cytokine levels in the supernatant of cocultures (IL-6, IL-17A, IL-17F, IFN- $\gamma$, IL-10, TGF- $\beta$, and IL-2) but did not observe any significant difference among the different conditions (data not shown). In vivo, we did not observe any difference in either $\mathrm{CD}^{+} \mathrm{CD} 25^{\text {hi }}$ Foxp $^{+}$Treg proportion among CD4 ${ }^{+} \mathrm{T}$ cells or in Treg absolute counts between HDs and SLE patients ( $n=10$ and $n=49$, respectively), regardless of disease activity (Supplemental Figure $3, \mathrm{~F}$ and G), thus ruling out an OX40L-dependent defect in Treg generation in human SLE. Foxp3 expression levels in blood Tregs were lower in SLE patients as compared with those in HDs (Figure 4E) and were negatively correlated with the proportion of OX40L-expressing CD11c $\mathrm{DR}^{+} \mathrm{DCs}$, thus strongly suggesting that OX40L-dependent Foxp3 downregulation also operates in vivo (Figure 4F). To gain further insight into the mechanisms leading to Foxp3 downregulation, we next measured IL-2 and IL-6 concentrations in the supernatants of GM-CSF IL-4/SLE DCs and Treg cocultures, as these cytokines have been shown to modulate Foxp3 expression (21-23). No difference in IL-2 production was detectable between culture conditions, whereas IL-6 concentration was higher in the presence of SLE DCs as compared with GM-CS$\mathrm{F}^{+} \mathrm{IL}-4$ DCs (data not shown). Taken together, these results show the critical role of aberrant OX40L-dependent signaling in Foxp3 downregulation associated with Treg dysfunction in patients with active SLE.

The OX40L/OX40 axis modulates follicular Treg functions. Tfr cells are a follicular subset critical for the regulation of the GC response through suppression of Tfh cell and B cell responses (24). Given that OX40L signaling affects Treg function, we hypothesized that OX40L signals may also effect the suppressive function of Tfr cells. In a first step, we purified human tonsil cells from HDs and studied extensive phenotyping of Tregs (Supplemental Figure 6A). Based on the expression of the chemokine receptor CXCR5, we were able to identify two populations of tonsil Foxp $3^{+}$Tregs. The first subset, $\mathrm{CD} 4^{+} \mathrm{CXCR} 5^{+} \mathrm{FoxP} 3^{+}$cells, also expressed CD25, GITR, PD-1, ICOS, and Bc1-6, which was consistent with the phenotype of Tfr cells 
A

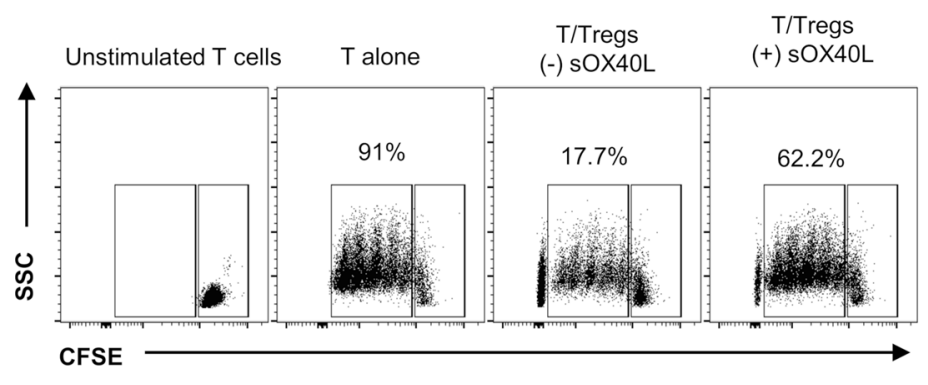

C

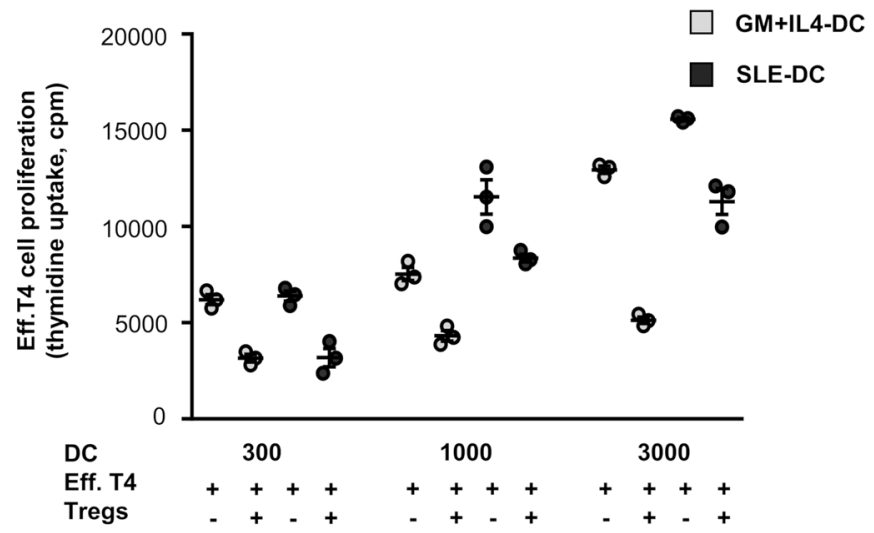

E

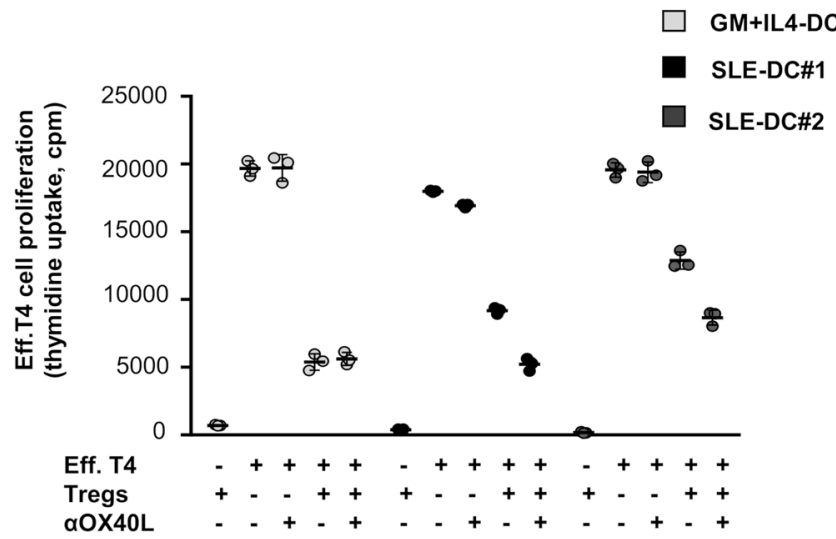

B

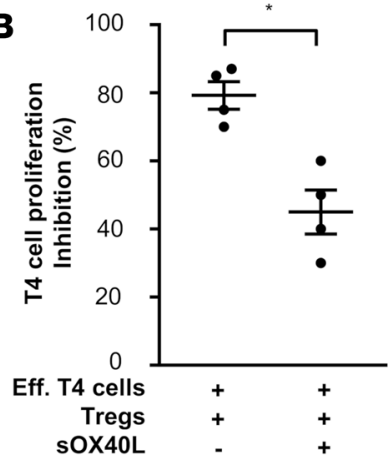

D

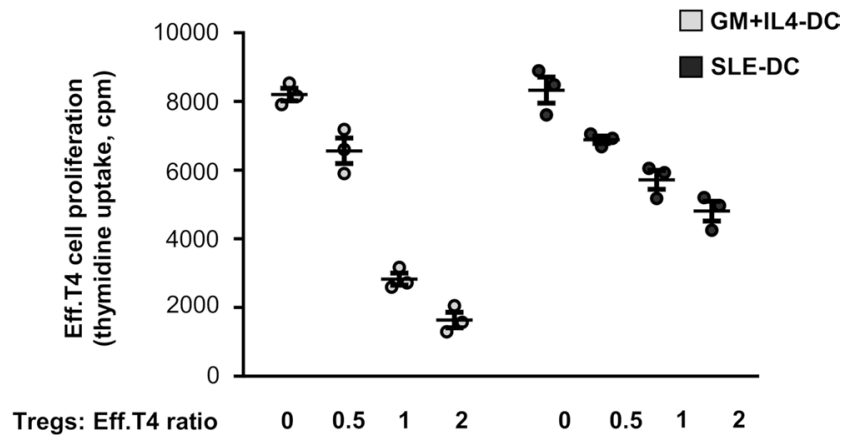

$\mathbf{F}$

GM+IL4-DC $(n=6)$

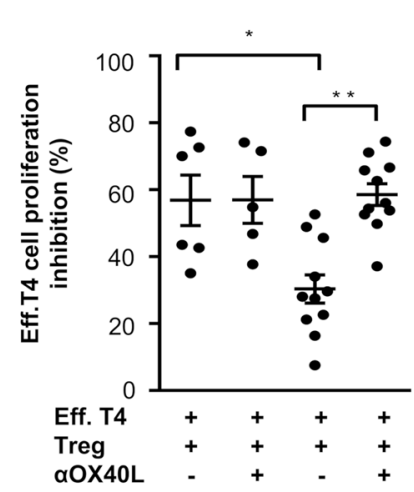

SLE-DC ( $n=10)$

Figure 1. OX40L impairs the suppressive function of Tregs. (A and B) Sorted effector T4 (Eff.T4) cells (10 ${ }^{4}$ cells) were labeled with CFSE (5 $\mu \mathrm{M}$ ), activated (anti-CD3, $1 \mu \mathrm{g} / \mathrm{ml}$ and anti-CD28, $3 \mu \mathrm{g} / \mathrm{ml}$ ) or not for unstimulated condition, and cultured for 3 days alone or with sorted Tregs (104 cells) in the presence or absence of soluble OX4OL (sOX40L) $(100 \mathrm{ng} / \mathrm{ml})$. Eff.T4 cell proliferation was assessed after 3 days of culture. (A) Representative dot plot showing proliferation (CFSE ${ }^{\text {dim }}$ ) of Eff.T4 cells after 3 days of culture. (B) Percentage of inhibition of Eff.T4 cell proliferation. The percentage of inhibition was calculated in reference to proliferation observed with stimulated Eff.T4 cells cultured alone. Error bars indicate the mean $\pm \mathrm{SEM}, n=4$ independent experiments. Statistical analysis was undertaken using the Mann-Whitney $U$ test. ${ }^{*} P<0.05$. (C-F) GM-CSF+IL-4 DCs or SLE DCs were cultured with purified Eff.T4 cells and Tregs for 3 days. Analysis of Eff.T4 cell proliferation was performed by (3H) thymidine incorporation measurement. (C) Analysis of Treg-suppressive function toward Eff.T4 cell proliferation at 3 different ratios of CM+IL-4 DCs or SLC DCs with Eff.T4 cells or Tregs (0.03:1:1, 0.1:1:1 and 0.3:1:1) of 3 independent experiments. (D) Analysis of Treg-suppressive function toward Eff.T4 cell proliferation at 4 different Treg/Eff.T4 cell ratios (0:1, 0.5:1, 1:1, and 2:1) of 3 independent experiments. (E) Representative experiment performed in triplicate showing that DCs, Tregs, and Eff.T4 cells were cocultured at a 0.1:1:1 ratio, respectively. Anti-OX40L blocking mAb restores Treg-suppressive function. (F) Cumulative data obtained with 6 CM-CSF+IL-4 DCs and 10 SLE DCs. GM-CS$\mathrm{F}^{+}$IL-4 DCs or SLE DCs, Eff.T4 cells, and Tregs were cultured at 0.1:1:1 ratio, respectively. Treg-suppressive function was defined as the percentage of Eff.T4 cell proliferation inhibition and calculated as follows: $\left.(\text { Eff.T4 }+ \text { Treg) })_{\text {condition cpm }} /(\text { Eff.T4) })_{\text {condition cpm }}\right) \times 100$. Statistical analysis was done using the Kruskal-Wallis test followed by Dunn's multiple comparison correction. ${ }^{*} P<0.05,{ }^{* *} P<0.002$.

(24). In contrast, the $\mathrm{CD} 4{ }^{+} \mathrm{CXCR} 5-\mathrm{FoxP}^{+}$subset did not express $\mathrm{Bcl}-6$ and $\mathrm{PD}-1$ and was defined as Tregs (Supplemental Figure 6A, right). In line with previous reports, Bcl-6 was downregulated in blood follicular counterparts $(13,14)$ (Supplemental Figure $6 \mathrm{~B}$ ). We also analyzed the frequency and the phenotype of the blood circulating counterparts of T follicular subsets in SLE patients (cTfh cells and cTfr cells). As compared 
A

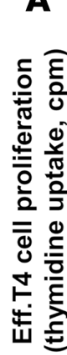

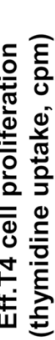

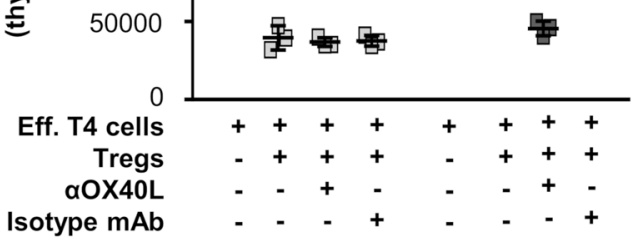

SLE APCs

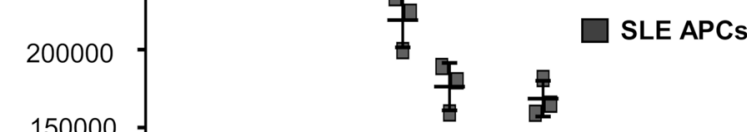

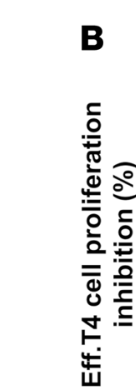

Eff. T4 cells

Tregs aOX40L

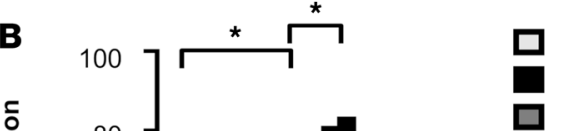

HD APCs $(n=7)$

aSLE APCs $(n=5)$

iSLE APCs $(n=5)$

\section{C}

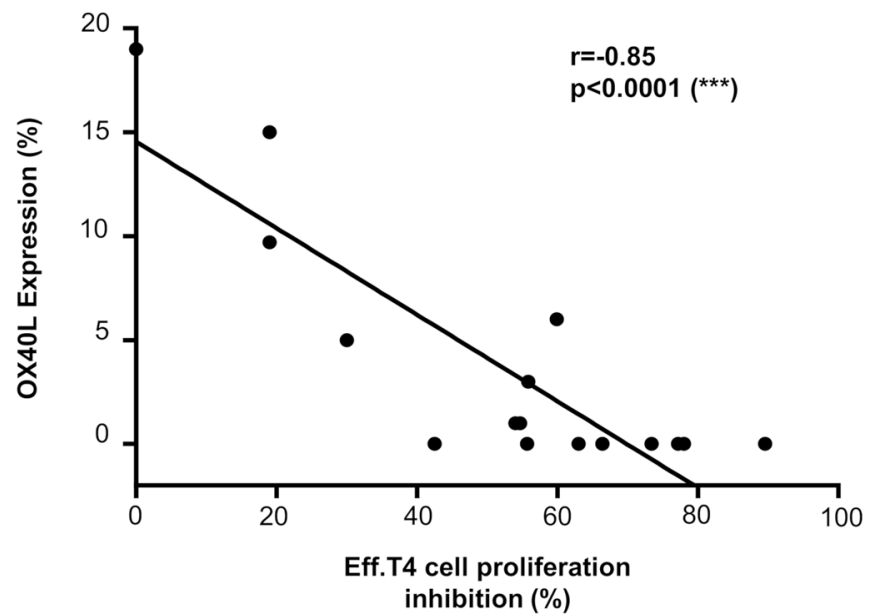

D

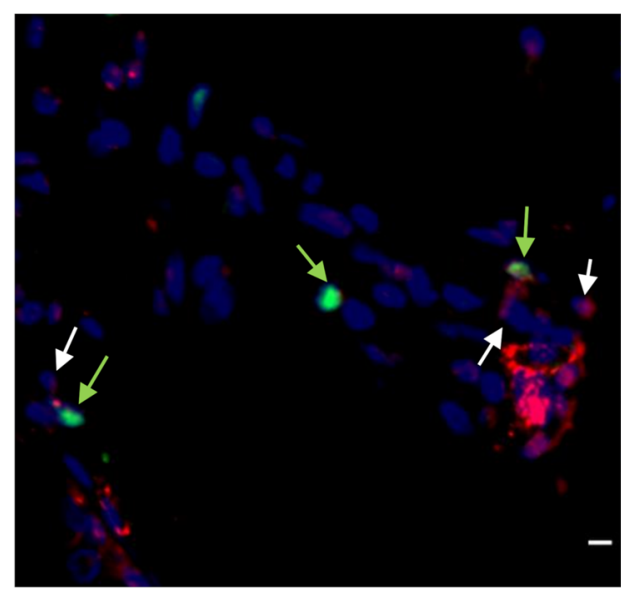

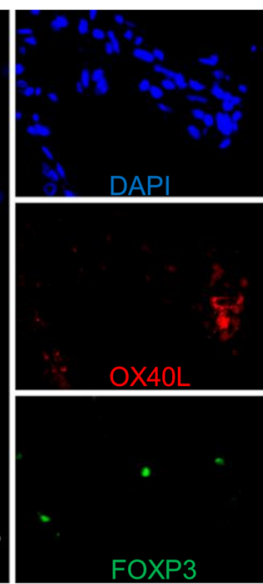

Figure 2. Circulating DCs from SLE patients suppress Treg function in an OX40L-dependent manner. (A-C) Blood-purified antigen-presenting cells (APCs) from active SLE (aSLE) patients, inactive SLE (iSLE) patients, or healthy donors (HD) were cultured in the presence of allogeneic Eff.T4 cells and Tregs at a 5:1:1 ratio, respectively, with or without blocking anti-OX40L mAb. An isotype control mouse IgG1 was used when indicated. Treg-suppressive function was calculated as described above. (A) This SLE patients' cells, used in this representative experiment, were clinically active, and his disease activity score (SLEDAI) was 16. (B) Cumulative data obtained from 5 aSLE (SLEDAI: 29, 4, 4 (clinical activity), 16, and 7, respectively) and 5 iSLE patients (SLEDAI: 0, 0, 0, 4 (biological activity only), 1, respectively) and 7 HD APCs. Statistical analyses were undertaken using the Kruskal-Wallis test followed by Dunn's multiple comparison correction. Error bars indicate the mean \pm SEM. (C) Correlation between Treg-suppressive function and circulating OX40L-expressing DCs. Statistical analysis was performed using Spearman's rank correlation test. (D) Skin biopsies from SLE patients were analyzed for OX4OL (in red) and Foxp3 (in green) expression by immunofluorescence. In a cell infiltrate, yellow arrowheads show Foxp3-expressing Tregs in close contact with $0 X 40 \mathrm{~L}$-expressing cells denoted by white arrowheads. Scale bar: $10 \mu \mathrm{m}$. Data are representative of 3 patients and controls. ${ }^{*} P<0.05,{ }^{* *} P<0.0001$.

with HDs and, in line with previous studies (17), we confirmed that SLE patients display a higher frequency of activated ICOS ${ }^{+} \mathrm{PD} 1^{+}$cTfh cells, which was positively correlated with $\mathrm{CD} 38^{+}$plasmablasts and SLEDAI. Regarding the Tfr counterpart, we did not observe any difference between HDs and SLE patients (Figure 5), and the disease activity did not affect the proportion of circulating Tfr cells as well.

Next, we analyzed the function of purified tonsil Tfr cells $\left(\mathrm{CD} 4^{+} \mathrm{CD} 25^{+} \mathrm{CXCR} 5^{+} \mathrm{ICOS}^{+}\right)$and $\mathrm{Tregs}$ $\left(\mathrm{CD} 4^{+} \mathrm{CD} 25^{+} \mathrm{CXCR} 5-\mathrm{ICOS}^{+}\right)$. Sorted cells of both subsets expressed Foxp3 (data not shown). When cultured with tonsil CFSE-labeled Tfh cells $\left(\mathrm{CD} 4^{+} \mathrm{CXCR}^{+}\right)$, both Tfr cells and Tregs were able to suppress the proliferation of Tfh cells. Preincubation of Tregs or Tfr cells with sOX40L resulted in a significant increase of Tfh cell proliferation (Figure 6, A and B), demonstrating that OX40L signal also affects the suppressive 
A

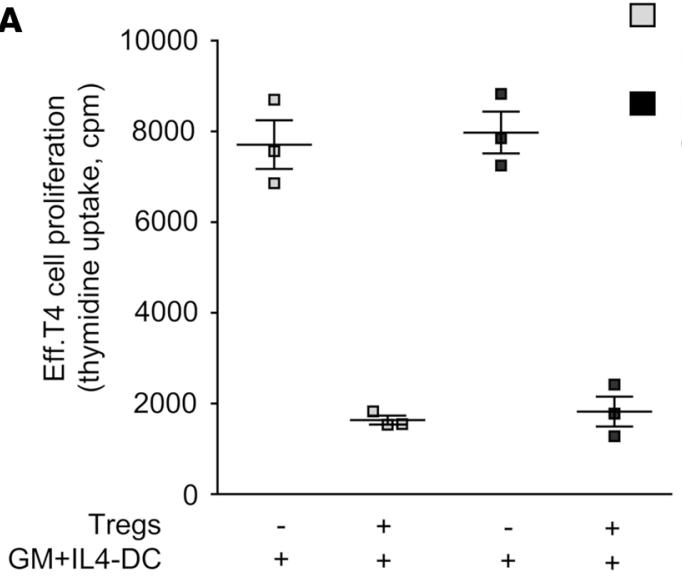

Eff. T4 from GM+IL4-DC Coculture

Eff. T4 from SLE-DC Coculture
B

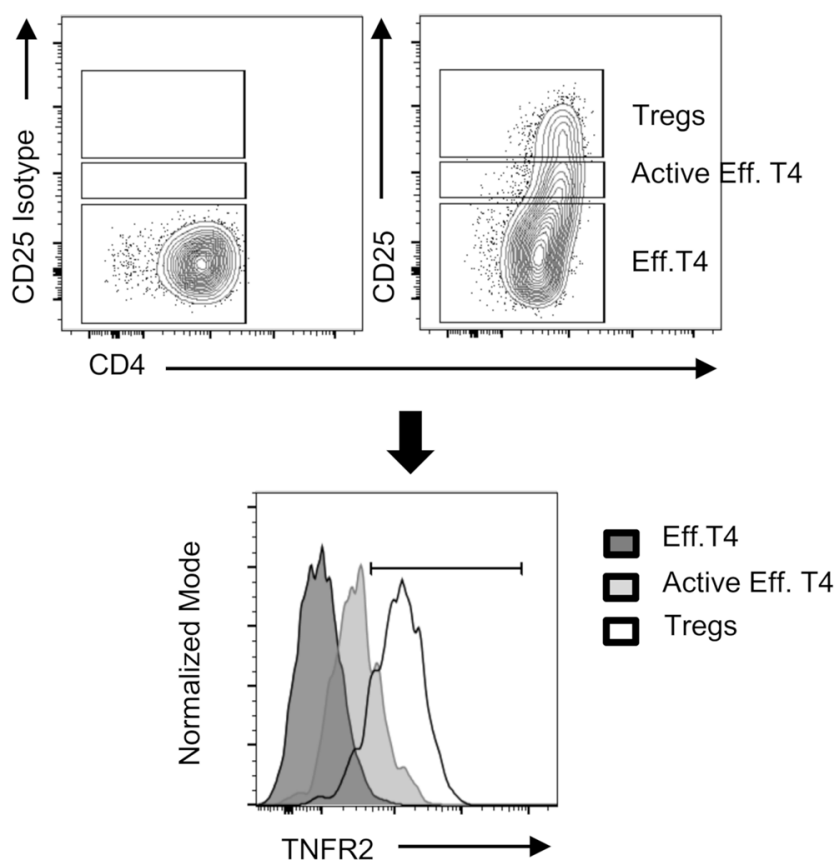

C

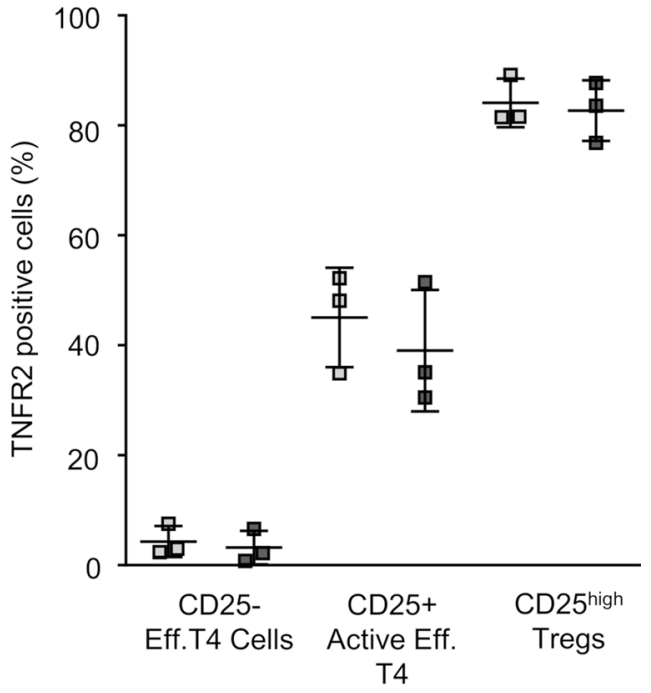

Figure 3. SLE DCs do not confer Tregs resistance to effector T4 cells. (A-C) Allogenic HD Eff.T4 cells $\left(5 \times 10^{4}\right)$ were cocultured either with GM-CSF+IL-4 DCs or SLE DCs $\left(5 \times 10^{3}\right)$ for 3 days. At 3 days, Eff.T4 cells were purified from culture and were further cocultured with GM-CSF+IL-4 DCs in the presence or absence of Tregs at a Eff.T4/Tregs/DC ratio of 1:1:0.1 respectively. (A) Analysis of Eff.T4 cell proliferation, performed by (3H) thymidine incorporation measurement. (B) Analysis of TNFR2 surface expression on CD25 Eff.T4 cells, CD25 active Eff.T4 cells, and CD25 hi Tregs from GM-CSF+IL-4 DC or SLE DC cocultures. Representative dot plot (top) and histogram (bottom) showing TNFR2 expression on CD25- Eff.T4 cells, CD25+ active Eff.T4 cells, and CD25 hi Tregs. (C) Cumulative data obtained from 3 cultures with purified T cells from CM-CSF+IL-4 or SLE DC cocultures represent the percentage of cells expressing surface TNFR2. Error bars indicate the mean $\pm \mathrm{SEM}, n=3$.

function of the follicular subset. We next evaluated whether the efficiency of Tfr cells in regulating B cell responses was also altered by OX40L signaling. Tfr cells previously cultured or not with sOX40L were cultured with purified Tfh cells and memory B cells in the presence of Staphylococcal Enterotoxin B (SEB). We observed a higher immunoglobulin production and an increased differentiation of B cells into CD $38^{+}$ plasmablasts in cocultures with Tfr cells exposed to sOX40L (Figure 6, C and D). Cytokine profiles of coculture supernatants revealed a marked decrease of TGF- $\beta$ when adding sOX40L (data not shown). Altogether, these data show that OX40L not only impairs the suppressive functions of Tregs, but also those of Tfr cells.

\section{Discussion}

Tregs represent a phenotypically and functionally heterogeneous group of lymphocytes that exert immunosuppressive activities on effector immune responses. Tregs play a key role in maintaining immune tolerance and 
A
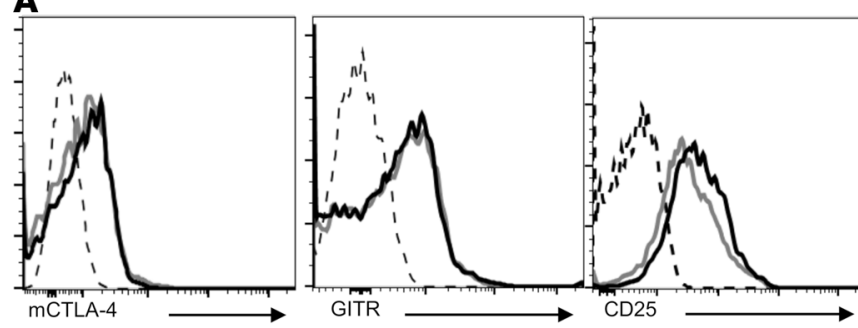

C

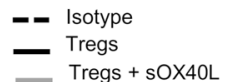

Tregs + sOX40L

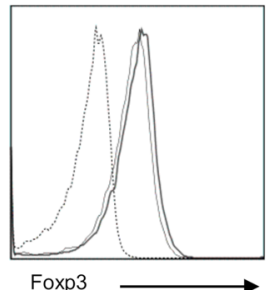

Foxp3

D

I:) Eff. T4/OX40L L Cells

$\square$ Treg/OX40L L Cells

$\square$ Treg/CD32 L Cells
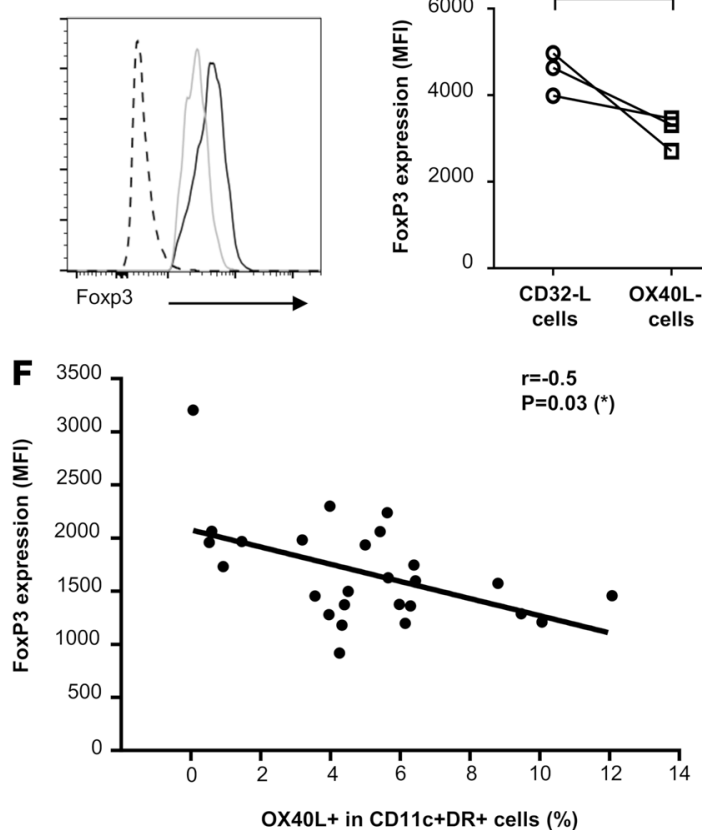

B
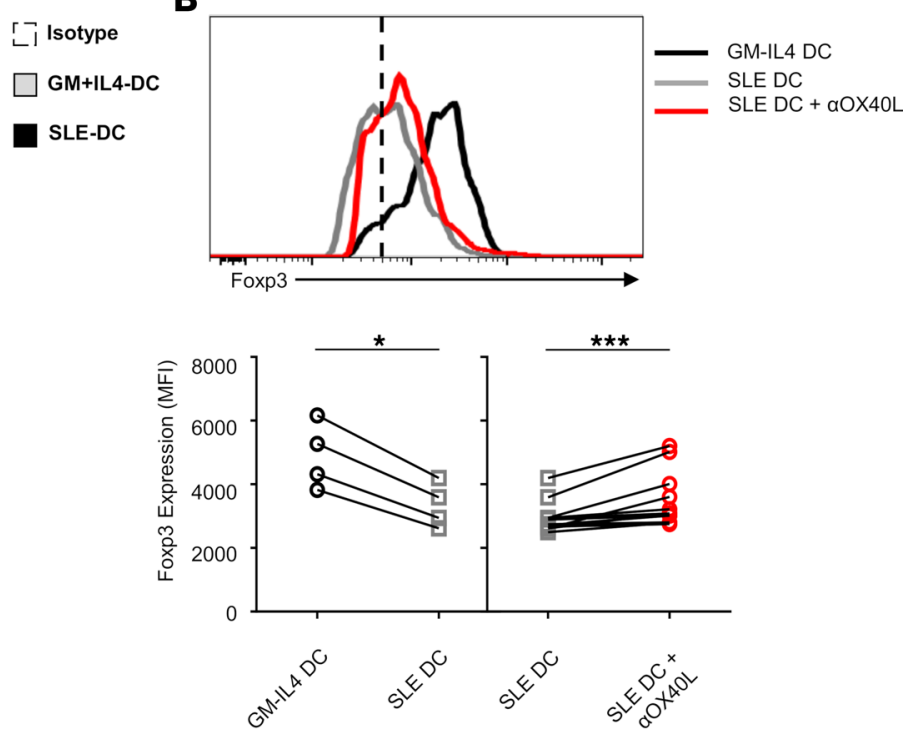
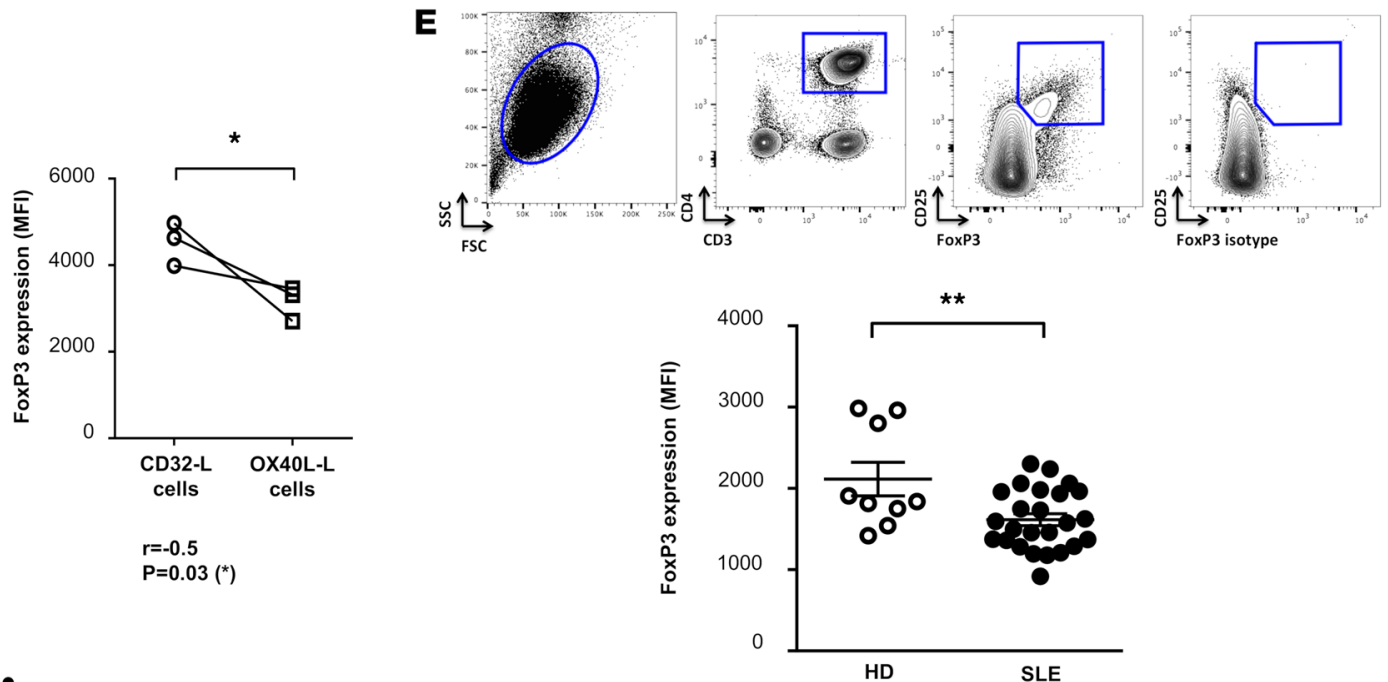

Figure 4. OX40L-dependent downregulation of Foxp3 expression in Tregs. (A and B) Tregs (10 ${ }^{4}$ cells) were cocultured in the presence or absence of anti-0X40L mAb $(10 \mu \mathrm{g} / \mathrm{ml})$ for 3 days with Eff.T4 cells ( $10^{4}$ cells) and either GM-CSF+IL-4 DCs or SLE DCs. (A) Representative histogram showing surface expression of CTLA-4, GITR, and CD25 on Foxp3+ Tregs. (B) Expression of Foxp3 in CD4+CD25 hiCD127- Tregs from cocultures with Eff.T4 cells and GM-CSF+IL-4 DCs or SLE DCs with or without anti-0X40L mAb. Representative histogram showing Foxp3 expression in Tregs cultured with GM-CSF+IL-4 DCs (black line), SLE DCs (gray line), and SLE DCs ${ }^{+}$anti-OX40L mAb (red line) (top). Cumulative data obtained with 4 different GM-CS$\mathrm{F}^{+}$IL-4 DCs and 13 different SLE DCs (bottom). Four independent experiments were undertaken and compared using nonparametric 2-tailed paired Wilcoxon test. ${ }^{*} P<0.05 ;{ }^{* *} P<0.001$. (C) Expression of Foxp3 in Tregs cultured with or without sOX40L (100 ng/ml). Representative histogram showing Foxp3 expression in Tregs cultured with (gray line) or without (black line) sOX40L (left). Dotted line represents Foxp3 isotype. Cumulative data from 5 independent experiments are shown and compared using nonparametric 2 -tailed Mann-Whitney test (right). ${ }^{*} P<0.05$. Results are expressed as Foxp3 MFI in Tregs. (D) Expression of Foxp3 in Eff.T4 cells and Tregs cultured for 3 days with OX40L or CD32-transfected L cells. Representative histogram showing Foxp3 expression in Eff.T4 cells (dashed line) and Tregs cultured with OX40L-transfected L cells (gray line) or CD32-tranfected L cells (black line) (left). Cumulative data from 3 independent experiments, compared using nonparametric 2-tailed paired Wilcoxon test (right). ${ }^{*} P<0.05$. Results are expressed as Foxp3 MFI in Tregs. (E) Foxp3 expression in CD3 ${ }^{+} C D 4{ }^{+} C D 25^{\text {hi }}$ blood cells of HDs and SLE patients. 
Representative dot plots showing blood $\mathrm{CD}^{+} \mathrm{CD}^{+} \mathrm{CD} 25^{\text {hi }} \mathrm{Foxp} 3^{+}$cells (top). Cumulative data analysis from 9 HDs and 25 SLE patients (bottom). Results are expressed as mean Foxp3 MFI in $\mathrm{CD}^{+} \mathrm{CD} 4{ }^{+} \mathrm{CD} 25^{\mathrm{hi}}$ cells. Error bars indicate the mean \pm SEM. (F) Correlation between proportion of blood OX40L-expressing CD11c ${ }^{+} D R^{+}$cells and Foxp3 MFI expression in CD3 ${ }^{+} C D 44^{+} C D 25^{\text {hi }}$ blood cells of SLE patients $(n=26)$. Statistical analysis was performed using Spearman's rank correlation test.

homeostasis through diverse mechanisms, which involve interactions with components of both the innate and adaptive immune systems (25). In SLE, as well as in many autoimmune diseases, Tregs have been proposed to play a relevant role in their pathogenesis. Initial studies regarding frequencies of Tregs in the peripheral blood of SLE patients have generated controversial results. Several groups observed a decreased proportion of $\mathrm{CD} 4{ }^{+} \mathrm{CD} 25^{\text {hi }}$ Tregs in SLE patients as compared with healthy controls and observed an inverse correlation of Treg numbers with disease activity $(8,9)$. However, other studies reported unaltered proportions or even increased proportions of Tregs in SLE patients and a positive correlation with disease activity (11). Our results did not reveal any quantitative defect in either the proportion or in absolute counts of circulating Tregs in SLE patients. Rather, our results identified an OX40L-dependent mechanism responsible for Treg dysfunction in inflammatory tissues. Indeed, we observed that myeloid cells expressing OX40L that migrated to inflammatory tissues in SLE patients colocalized with Tregs and OX40L impairs in vitro immunosuppressive properties of Tregs. Although we cannot rule out a local upregulation of OX40L by myeloid APCs within the tissues, these results established the OX40L/OX40 axis as critical mechanism in explaining dysregulated suppressive functions in the SLE inflammatory microenvironment.

Tregs have been shown to downregulate FoxP3 expression and to potentially revert to or act as effector cells. These cells promote inflammation by the production of inflammatory cytokines, such as IFN- $\gamma$ or IL-17 $(26,27)$. It was first suggested that these ex Tregs develop prior to Treg commitment, but a recent study has demonstrated that a fraction of bona fide Tregs with a demethylated TSDR in the Foxp3 locus also downregulates Foxp3 during an inflammatory autoimmune response and acquires effector T cell functions (28). In SLE patients, Foxp3 expression is consistently decreased, possibly due to IL-2 deficiency, in addition to other possible mechanisms (29). In this regard, our results demonstrate that OX40L-dependent signaling on Tregs could be another mechanism explaining Foxp3 downregulation in SLE patients. Interestingly, several authors have demonstrated in mouse models the ability of OX40 agonists to drive Treg expansion $(30,31)$. This discrepancy may rely on the cytokine microenvironment and the timing of the signal. As an example, OX40 agonist administration influenced experimental autoimmune encephalomyelitis disease severity in opposite directions, depending on the timing of administration (30). The mechanisms and the precise intracytoplasmic pathway by which OX40L hampers Treg functions and Foxp3 expression remain to be discovered.

Regulation of (auto)antibody production primarily takes place in the GC, and an aberrant GC response can contribute to autoimmunity (32). In this GC process, Tfh cells select B cells based on their capacity to bind and present the specific antigen (33). The recently identified Tfr cells are ideal candidates for regulating the normal GC response and preventing emergence of autoreactive B cells. Tfr cells constitute $5 \%-25 \%$ of the GC T cells in mice and originate from Foxp $3^{+}$thymic-derived Treg precursors. In the absence of Tfr cells, overwhelming outgrowth of nonspecific B cells leads to lower amounts of antigen-specific B cells $(13,14)$. Although of interest, no report has addressed the possible role of Tfr cells in the pathogenesis of SLE. Interestingly, based on the evaluation of the circulating $\mathrm{CD} 4{ }^{+} \mathrm{CXCR} 5^{+} \mathrm{CD} 25^{\mathrm{hi}} \mathrm{CD} 127^{-} \mathrm{Foxp} 3^{+}$cells, we could not detect any quantitative defect related to diseases activity in SLE patients. Rather, our results implicate Tfr cells in pathogenesis through their inability to block GC under inflammatory conditions. Our previous work showed that upon TLR7-dependent triggering signals, OX40L is upregulated on myeloid APCs (16) within the inflammatory tissues. This set of data provides evidence that OX40L-deregulated signals block the suppressive functions of Tfr cells, without altering their ability to migrate into the B cell area.

Altogether, our observations identify the OX40L/OX40 axis as an important enhancing inflammatory loop in SLE patients, as it can promote the differentiation of naive and memory $\mathrm{T}$ cells into follicular T lymphocytes while blocking the suppressive function of Tregs and Tfr cells. Therefore, blocking of the OX40L/OX40 axis should be considered as a new target option for future clinical trials in lupus.

\section{Methods}

Patients. Sixty-one consecutive SLE patients who met the revised criteria of the American College of Rheumatology for SLE (18) were enrolled. All clinically and biologically relevant information concerning the patients is provided in Supplemental Table 1. The active disease patient group was defined as having 
A
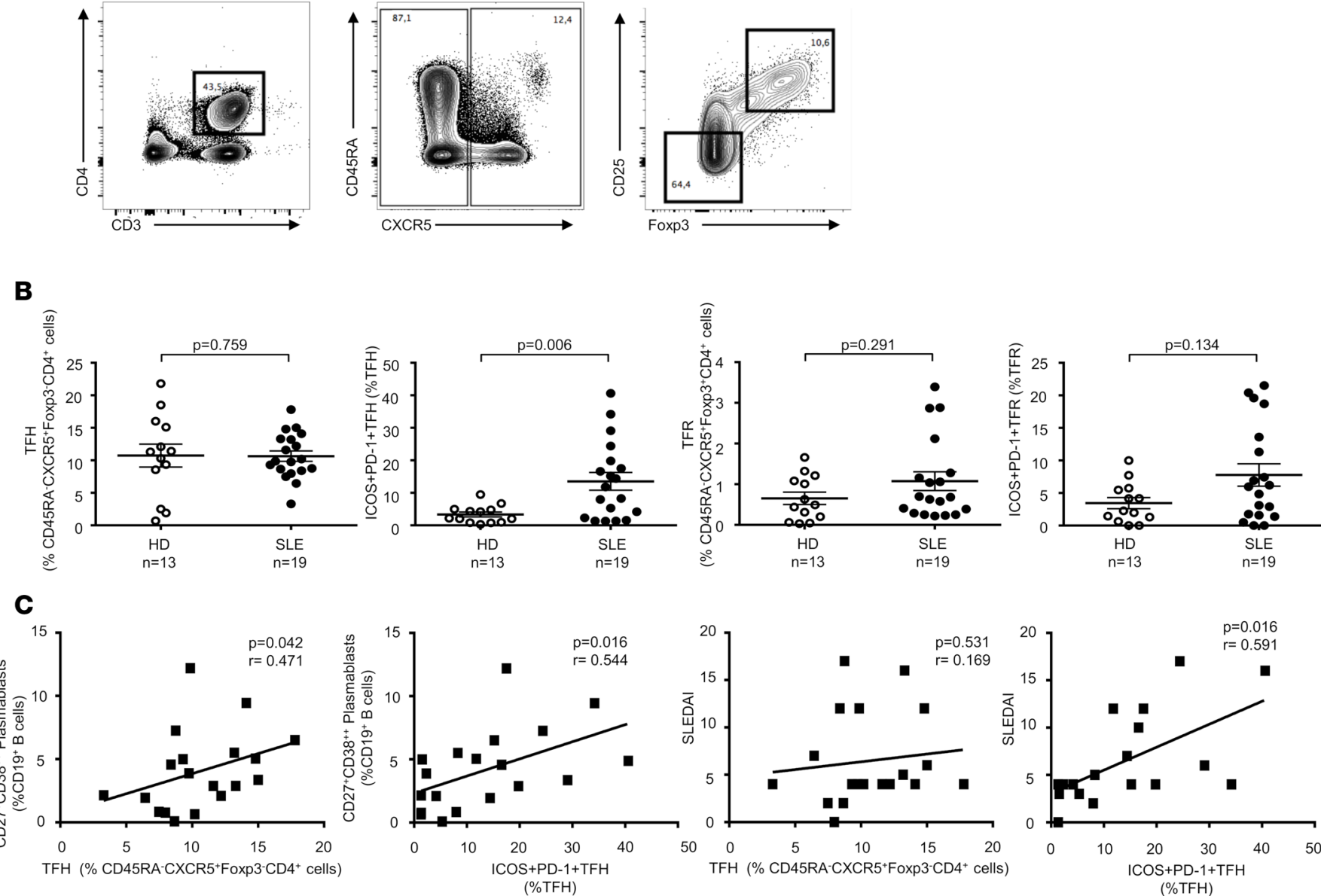

Figure 5. The association of blood Tfh and Tfr cells in HDs and SLE patients with disease activity and plasmablast frequency. (A) Representative dot plots showing the gating strategy. (B) Frequency of Tfh cells (CD4+CD45RA-CXCR5+FoxP3-), Tfr cells (CD4+CD45RA-CXCR5 $5^{+}$FoxP3 ${ }^{+}$), activated Tfh cells (aTfh cell, PD1+ICOS $)$, and activated Tfr cells (aTfr, PD1+ICOS+) in SLE patients $(n=19)$ and in HDs $(n=13)$. Statistical analysis was conducted using the 2-tailed Mann-Whitney $U$ test. Error bars indicate the mean \pm SEM. (C) Correlation between Tfh cell subsets, plasmablasts $\left(\right.$ CD27 ${ }^{+}$CD38 $8^{+}$CD19 ${ }^{+}$), and SLEDAI. Statistical analysis was conducted using the Spearman's rank correlation test.

a SLEDAI score of $\geq 6$. HDs from our staff (13 females and 3 males, all provided informed consent, approved by the ethics committee of Bordeaux University Hospital) were studied as a control group.

Phenotypic analysis of blood samples. Whole blood analysis of OX40L expression was conducted using anti-human CD14, CD16, CD11c, HLA-DR, and OX40L mAbs incubated in whole blood following red blood cells lysis (Versalyse solution from Beckman Coulter). For Treg analysis, cells were stained with anti-CD3, CD4, and CD25, before intranuclear staining for Foxp3 according to the manufacturer's instructions (eBioscience Foxp3/Transcription Factor Staining Buffer Set). Only the CD25 hi Foxp3 ${ }^{+}$cells were analyzed. For Tfr cell analysis, the following antibody panel was used: anti-CD3, CD4, CD25, ICOS, CD45RA, PD1, and CXCR5; this was followed by intranuclear staining for Foxp3. Data were collected using a BD FACSCanto II or a BD Fortessa and analyzed with DIVA and Flowjo softwares (BD Biosciences and Tree Star, respectively). All antibodies used in this study are listed in Supplemental Table 2.

Phenotypic analysis of tonsil samples. Tonsil samples were obtained from HDs undergoing tonsillectomies, and single cells were collected by mechanical disruption. For surface staining, cells were incubated with fluorochrome-conjugated antibodies: anti-CD4, -CD3, and -CD25 from Beckman Coulter; PD-1, ICOS, and GITR from Biolegend for 15 minutes; followed by intranuclear staining with Foxp3 and Bcl-6. Samples were analyzed using a BD Fortessa and analyzed with DIVA and Flowjo softwares (BD Biosciences and Tree Star, respectively).

Immunofluorescence experiments. Punch biopsy specimens (4 $\mathrm{mm}$ ) of affected skin were obtained from 3 SLE patients. Immunofluorescence was performed as previously described by Truchetet ME, et al. (34). 
A

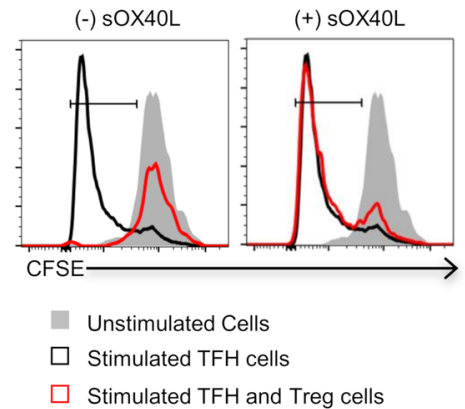

Treg

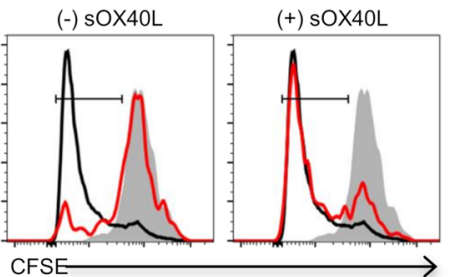

B

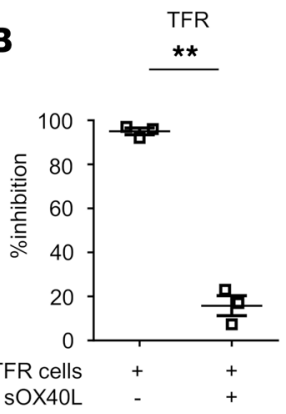

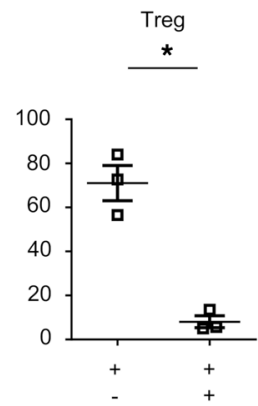

C TFH:LB:TFR (1:1:2)

TFH:LB:Treg (1:1:2)
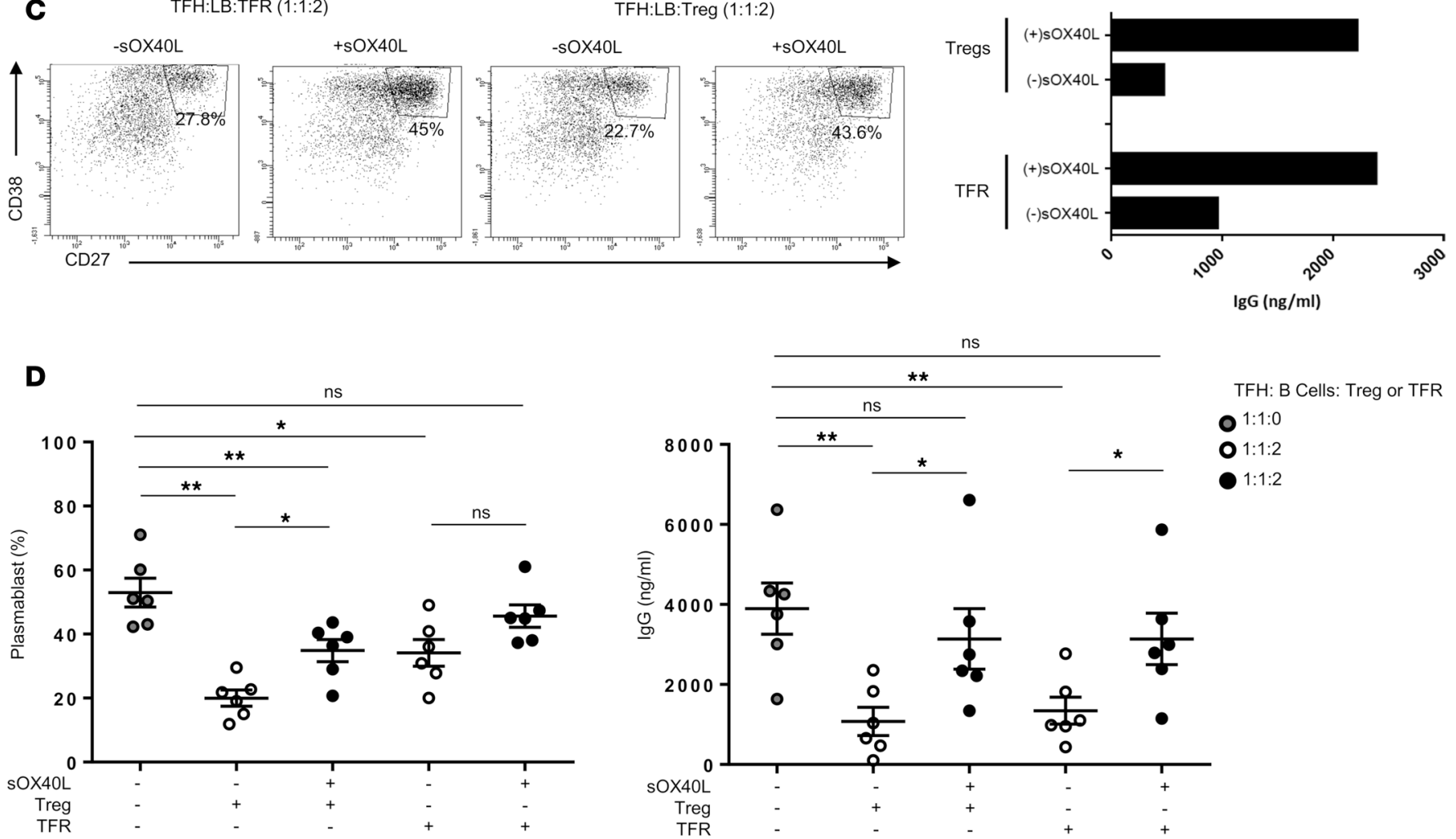

Figure 6. 0X40-0X40L axis modulates Tfr cells regulatory functions. (A and B) Sorted tonsil CD4+CD25-ICOS+CXCR5 ${ }^{+}$Tfh cells (10 ${ }^{4}$ cells) were either stimulated (CD3 and CD28 mAb) or not and were cultured for 3 days alone or with sorted tonsil CD4 $4^{+}$CD25 ICOS ${ }^{+}$CXCR5 $5^{+}$Tfr cells (10 ${ }^{4}$ cells) or CD4+CD25+ICOS+CXCR5- Tregs ( $10^{4}$ cells) in the presence or absence of sOX4OL $(100 \mathrm{ng} / \mathrm{ml})$. Proliferation of Tfh cells was analyzed at 3 days of coculture. (A) Histograms of a representative experiment showing proliferation (CFSE ${ }^{\mathrm{dim}}$ ) of unstimulated Tfh cells (gray filled), stimulated Tfh cells (black line), and Tfh cells cultured with either Tfr cells or Tregs (red line) at 3 days of culture. (B) Percentage inhibition of Tfh cell proliferation. The percentage of inhibition was calculated in reference to proliferation observed with stimulated Tfh cells cultured alone. Error bars indicate the mean \pm SEM, $n=3$. Statistical analysis was conducted using the 1-way ANOVA test. (C and D) Soluble OX40L impairs the ability of Tfr cells and Tregs to suppress Tfh cell function. After incubation with sOX40L $(100 \mathrm{ng} / \mathrm{ml})$, Tregs or Tfr cells $\left(4 \times 10^{4}\right.$ cells) were cultured for 7 days along with Tfh cells $\left(2 \times 10^{4}\right)$ and memory B cells $\left(2 \times 10^{4}\right)$ in the presence of SEB $(0.25 \mathrm{ng} / \mathrm{ml})$. CD27+CD38 $8^{+}$plasmablast percentage in cell culture (C) and concentration of IgC in cell culture supernatant (D) were determined by flow cytometry and ELISA, respectively, after 7 days of coculture. Individual values are shown with mean and SEM and were compared using nonparametric Kruskal-Wallis test with Dunn's comparison for multiple comparisons. ${ }^{*} P<0.05$; ${ }^{* *} P<0.01$.

Monoclonal mouse anti-human OX40L (clone 159403, mouse IgG1) was from R\&D Systems, rat anti-human FoxP3 (clone PCH101, rat IgG2א) was from eBioscience, while Alexa Fluor 488-conjugated donkey anti-rat and Alexa Fluor 547-conjugated donkey anti-mouse were from Invitrogen. After paraffin removal, epitope retrieval, and blocking in PBS-4\% BSA, tissue sections were incubated with anti-FoxP3 and anti-OX40L. Binding was revealed with Alexa Fluor 488- or Alexa Fluor 547-conjugated anti-rat or anti-mouse sera. Nuclei were stained with DAPI. Laser-scanning confocal images were acquired using a 
Zeiss LSM 510 META confocal laser-scanning microscope (Carl Zeiss). Negative controls, stained only with secondary antibodies, did not result in significant fluorescence and were omitted from the figures.

Monocyte-derived DC generation and purification of DCs from patients and HDs. Monocyte-derived DC preparations were generated as previously described (3). Briefly, CD14+ monocyte cells were purified from PBMCs using anti-CD14 MicroBeads (Miltenyi Biotec). $25 \mathrm{ng} / \mathrm{ml}$ recombinant human GM-CSF and $10 \mathrm{ng} / \mathrm{ml}$ recombinant human IL-4 purchased from PeproTech were add to $\mathrm{CD} 14^{+}$purified monocytes in complete RPMI 1640 medium (supplemented with 8\% FCS). At day 4, DCs were collected and analyzed by flow cytometry to determine the expression levels of CD14, HLA-DR, CD11c, and OX40L. For ex vivo experiments, the non-T cell fractions were negatively isolated from HDs or the peripheral blood mononuclear cells (PBMCs) of SLE patients using anti-CD3 MicroBeads (Miltenyi Biotec), and the cells were irradiated (35 Gy).

Effector T cell and Treg purification. Eff.T4 cells and Tregs were isolated from HD PBMCs by magnetic cell separation using CD4 MicroBeads (Miltenyi Biotec). After 18 hours at $4^{\circ} \mathrm{C}$, the effluent preenriched $\mathrm{CD}^{+} \mathrm{T}$ cells were labeled with anti-CD127 antibody and coupled with anti-Mouse IgG MicroBeads (Miltenyi Biotec). The negative fraction was labeled with CD25 MicroBead II (Miltenyi Biotec) to obtain $\mathrm{CD} 4{ }^{+} \mathrm{CD} 127^{-/ 10} \mathrm{CD} 25^{+}$Tregs. Naive T cells were purified from preenriched $\mathrm{CD} 4^{+} \mathrm{T}$ cells by sorting with FACSAria (BD Biosciences). Purity was controlled by flow cytometry analysis using LIVE/ DEAD FIXABLE Aqua (Invitrogen) and was always above 95\%.

Treg functional assays. For $\left({ }^{3} \mathrm{H}\right)$ thymidine-based suppression assays, freshly isolated Tregs $\left(10^{4}\right.$ cells $)$ and Eff.T4 cells $\left(10^{4}\right.$ cells) were cultured in complete RPMI 1640 medium (supplemented with 8\% FCS) in 96-well round-bottomed microplates (Becton Dickinson) with $1 \mu \mathrm{g} / \mathrm{ml}$ plate-bound anti-CD3 and SLE or GM-CS$\mathrm{F}^{+} \mathrm{IL}-4 \mathrm{DCs}\left(10^{3} \mathrm{cells}\right)$ with or without $10 \mu \mathrm{g} / \mathrm{ml}$ blocking anti-OX40L mAbs (Ancell) or isotype-matched mAbs. For APC-based suppression assay, Tregs $\left(10^{4} \mathrm{cells}\right)$ and Eff.T4 cells $\left(10^{4}\right.$ cells $)$ were then cultured together with irradiated allogenic (35 Gy) CD3 ${ }^{+}$cells depleted PBMCs (APCs) $\left(5 \times 10^{3}\right.$ cells). After 3 days of culture, $\left({ }^{3} \mathrm{H}\right)$ thymidine $(1 \mu \mathrm{Ci})$ was added for 18 additional hours of culture. Proliferation was determined using a scintillation counter (Microbeta Trilux, Wallac). All clinically and biologically relevant information concerning the patients included in the Treg functional assay are provided in Supplemental Table 3.

Th and B cell coculture. Tfh cells, Tregs, and Tfr cells were purified from preenriched CD4 ${ }^{+} \mathrm{T}$ cells and sorted with a FACSAria. Autologous memory LB were purified from preenriched CD19+ cells and sorted with a FACSAria $\left(\mathrm{CD}^{-} \mathrm{CD} 19^{+} \mathrm{CD} 27^{+} \mathrm{IgD}^{-}\right)$. Tfr cells or Tregs were incubated with or without $100 \mathrm{ng} / \mathrm{ml}$ sOX40L $\left(2 \times 10^{4}\right.$ or $\left.4 \times 10^{4}\right)$ during one in complete RPMI medium. After 1 hour the cells were washed and cocultured with Tfh cells and memory LB in complete RPMI medium in the presence of endotoxin-reduced SEB (Toxin Technology). The percentage of plasmablast was analyzed by flow cytometry, and IgG produced in supernatants was analyzed by ELISA at day 7.

Analysis of Foxp3 expression. Purified Tregs and/or Eff.T4 cells $\left(5 \times 10^{4}\right.$ cells) were cultured with or without of sOX40L $(100 \mathrm{ng} / \mathrm{ml})$ or cocultured with SLE or GM-CSF ${ }^{+} \mathrm{IL}_{-} 4 \mathrm{DCs}\left(5 \times 10^{3}\right.$ cells $)$ in $96-$-well round-bottom plates precoated with $1 \mu \mathrm{g} / \mathrm{ml}$ anti-CD3. After 48 hours of culture, cells were incubated with fluorochrome-conjugated antibodies CD4, CD3, CD25, CTLA-4, and GITR. After surface staining, cells were fixed, permeabilized, and stained for Foxp3 per the manufacturer's instructions (ebioscience, Foxp3/ Transcription Factor Staining Buffer Set).

Irradiated (60 Gy) CD32-expressing L cells or OX40L-expressing L cells were cultured in RPMI complete medium for 3 days with Eff.T4 cells or Tregs (2.5:1 for T cells/L cell ratio) in 96-well round-bottom plates precoated with $1 \mu \mathrm{g} / \mathrm{ml}$ anti-CD3. After 3 days, cells were collected, surface stained with anti-CD4 and -CD25, and intranuclear stained for Foxp3.

sOX40L and cytokines measurements. sOX40L was measured by ELISA (Cusabio) in sera of SLE patients and HDs, in accordance with the manufacturer's recommendations. Cytokines were measured using Cytometric Bead Array from Biolegend (Legendplex).

Statistics. The normality of the variable distribution was assessed using the Kruskal-Wallis test. In case of normality of data distribution, 2-tailed Student's $t$ tests were performed, or in case of more than two groups, a 1-way ANOVA test with Holm-Sidak's correction for multiple comparisons was used. When the normality of the distribution was rejected, statistical analyses were performed using the nonparametric paired Wilcoxon test or unpaired Mann-Whitney $U$ test as appropriate or with Kruskal-Wallis followed by Dunn's correction for multiple comparisons. Correlation between variables was determined using the Spearman test. $P<0.05$ was considered statistically significant. All statistical analyses were performed using GraphPad Prism 6.0 software (SAS Institute). 
Study approval. The study protocol was approved by the ethics committee of Bordeaux University Hospital. Each patient enrolled in the study signed a consent form approved by the ethics committee.

\section{Author contributions}

CJ, JFA, ID, CCB, and MS analyzed the phenotype and performed statistical analysis of blood samples. CJ, ID, JFA, and CCB performed the in vitro experiments with DCs. MET, EL, and JS performed tissue staining. ID, JFA, CCB, and EF performed the in vitro experiments with effector, Trf, and B cells and ELISA. CCB was involved in the experimental design. CR, EL, PD, MET, LC, TS, JFV, and JLP provided adult SLE samples and clinical information. MS performed Helios staining and multiplex cytokine assays. VP was involved in the design of the experiments and in the providing of SLE patient samples with clinical information. NG was involved in performing in vitro DC experiments and phenotyping analysis of blood samples. Data interpretation was performed by all authors. CJ, JFA, and PB wrote the manuscript. $\mathrm{PB}$ conceived the project and oversaw the entire work.

\section{Acknowledgments}

We thank J.-C. Caron, K. Eschel, M. Gassie, and C. Cognet for technical help provided for flow cytometry. We thank V. Pitard and V. De Luca for cell sorting. We thank H. Ueno and V. Pascual for discussions. This study was supported by research funding from Centre national pour la Recherche Scientifique, the Société Française de Rhumatologie, Société française Nationale de Médecine Interne, Arthritis Fondation Courtin, Ministère de la Recherche et de l'Enseignement supérieur, the NIH (grants U19-AI057234, U19-AI082715, U19-AI089987), Alliance for Lupus Research, and Baylor Health Care System.

Address correspondence to: Patrick Blanco, CNRS-UMR 5164 Immuno ConcEpT, 146, rue Léo-Saignat, 33076 Bordeaux, France. Phone: 33.557571472. Email: patrick.blanco@chu-bordeaux.fr.

1. Tsokos GC. Systemic lupus erythematosus. N Engl J Med. 2011;365(22):2110-2121.

2. Franklyn K, Hoi A, Nikpour M, Morand EF. The need to define treatment goals for systemic lupus erythematosus. Nat Rev Rheumatol. 2014;10(9):567-571.

3. Blanco P, Palucka AK, Gill M, Pascual V, Banchereau J. Induction of dendritic cell differentiation by IFN-alpha in systemic lupus erythematosus. Science. 2001;294(5546):1540-1543.

4. Blanco P, Palucka AK, Pascual V, Banchereau J. Dendritic cells and cytokines in human inflammatory and autoimmune diseases. Cytokine Growth Factor Rev. 2008;19(1):41-52.

5. Campbell DJ, Koch MA. Phenotypical and functional specialization of FOXP3+ regulatory T cells. Nat Rev Immunol. 2011;11(2):119-130.

6. Scalapino KJ, Tang Q, Bluestone JA, Bonyhadi ML, Daikh DI. Suppression of disease in New Zealand Black/New Zealand White lupus-prone mice by adoptive transfer of ex vivo expanded regulatory T cells. J Immunol. 2006;177(3):1451-1459.

7. Ohl K, Tenbrock K. Regulatory T cells in systemic lupus erythematosus. Eur J Immunol. 2015;45(2):344-355.

8. Valencia X, Yarboro C, Illei G, Lipsky PE. Deficient CD4+CD25high T regulatory cell function in patients with active systemic lupus erythematosus. J Immunol. 2007;178(4):2579-2588.

9. Miyara M, et al. Global natural regulatory $\mathrm{T}$ cell depletion in active systemic lupus erythematosus. J Immunol. 2005;175(12):8392-8400.

10. Alvarado-Sánchez B, et al. Regulatory T cells in patients with systemic lupus erythematosus. J Autoimmun. 2006;27(2):110-118.

11. Golding A, Hasni S, Illei G, Shevach EM. The percentage of FoxP3+Helios+ Treg cells correlates positively with disease activity in systemic lupus erythematosus. Arthritis Rheum. 2013;65(11):2898-2906.

12. Alexander T, et al. Foxp3+ Helios+ regulatory T cells are expanded in active systemic lupus erythematosus. Ann Rheum Dis. 2013;72(9):1549-1558

13. Linterman MA, et al. Foxp3+ follicular regulatory T cells control the germinal center response. Nat Med. 2011;17(8):975-982.

14. Chung Y, et al. Follicular regulatory T cells expressing Foxp3 and Bcl-6 suppress germinal center reactions. Nat Med. 2011;17(8):983-988.

15. Grammer AC, et al. Abnormal germinal center reactions in systemic lupus erythematosus demonstrated by blockade of CD154CD40 interactions. J Clin Invest. 2003;112(10):1506-1520.

16. Jacquemin C, et al. OX40 ligand contributes to human lupus pathogenesis by promoting $\mathrm{T}$ follicular helper response. Immunity. 2015;42(6):1159-1170.

17. Blanco P, Ueno H, Schmitt N. T follicular helper (Tfh) cells in lupus: activation and involvement in SLE pathogenesis. Eur $J$ Immunol. 2016;46(2):281-290.

18. Voo KS, et al. Antibodies targeting human OX40 expand effector T cells and block inducible and natural regulatory T cell function. J Immunol. 2013;191(7):3641-3650.

19. Venigalla RK, et al. Reduced CD4+,CD25- T cell sensitivity to the suppressive function of CD4+,CD25high,CD127 -/low regulatory T cells in patients with active systemic lupus erythematosus. Arthritis Rheum. 2008;58(7):2120-2130.

20. Chen X, Hamano R, Subleski JJ, Hurwitz AA, Howard OM, Oppenheim JJ. Expression of costimulatory TNFR2 
induces resistance of CD4+FoxP3- conventional T cells to suppression by CD4+FoxP3+ regulatory T cells. J Immunol. 2010;185(1):174-182

21. Humrich JY, et al. Homeostatic imbalance of regulatory and effector T cells due to IL-2 deprivation amplifies murine lupus. Proc Natl Acad Sci USA. 2010;107(1):204-209.

22. Kastner L, Dwyer D, Qin FX. Synergistic effect of IL-6 and IL-4 in driving fate revision of natural Foxp3+ regulatory T cells. J Immunol. 2010;185(10):5778-5786.

23. Rubtsov YP, et al. Stability of the regulatory T cell lineage in vivo. Science. 2010;329(5999):1667-1671.

24. Sage PT, Sharpe AH. T follicular regulatory cells in the regulation of B cell responses. Trends Immunol. 2015;36(7):410-418.

25. Sakaguchi S, Vignali DA, Rudensky AY, Niec RE, Waldmann H. The plasticity and stability of regulatory T cells. Nat Rev Immunol. 2013;13(6):461-467.

26. Komatsu N, et al. Pathogenic conversion of Foxp3+ T cells into TH17 cells in autoimmune arthritis. Nat Med. 2014;20(1):62-68.

27. Zhou X, et al. Instability of the transcription factor Foxp3 leads to the generation of pathogenic memory T cells in vivo. Nat Immunol. 2009;10(9):1000-1007.

28. Spence A, Klementowicz JE, Bluestone JA, Tang Q. Targeting Treg signaling for the treatment of autoimmune diseases. Curr Opin Immunol. 2015;37:11-20.

29. Comte D, et al. Engagement of SLAMF3 enhances CD4+ T-cell sensitivity to IL-2 and favors regulatory T-cell polarization in systemic lupus erythematosus. Proc Natl Acad Sci USA. 2016;113(33):9321-9326.

30. Ruby CE, et al. Cutting Edge: OX40 agonists can drive regulatory T cell expansion if the cytokine milieu is right. J Immunol. 2009;183(8):4853-4857.

31. Gopisetty A, et al. OX40L/Jagged1 cosignaling by GM-CSF-induced bone marrow-derived dendritic cells is required for the expansion of functional regulatory T cells. J Immunol. 2013;190(11):5516-5525.

32. Ueno H. T follicular helper cells in human autoimmunity. Curr Opin Immunol. 2016;43:24-31.

33. Schmitt N, Ueno H. Human T follicular helper cells: development and subsets. Adv Exp Med Biol. 2013;785:87-94

34. Truchetet ME, et al. Platelets induce thymic stromal lymphopoietin production by endothelial cells: contribution to fibrosis in human systemic sclerosis. Arthritis Rheumatol. 2016;68(11):2784-2794. 